\title{
Construct freeform surface directly in an initial layout of an off-axis reflective image system by seed curve extension and simulated annealing algorithm
}

\author{
Hongxiang Pan ${ }^{1}$, Zhouping Su, ${ }^{1,2^{*}}$, Yangliu Zhang ${ }^{1}$ and Tao $\mathrm{Wu}^{1}$
}

\begin{abstract}
In this paper, a method of how to construct a freeform surface directly in an off-axis reflective image system is proposed. The method includes both the seed curve extension algorithm and simulated annealing algorithm. Firstly, the sample points on the unkown freeform surface were be obtained quickly by the seed curve extension algorithm. Then the continuity of the freeform surface is evaluated by calculating the angle between the normal vectors at the adjacent sample points. At last the freeform surface was fitted to an extended polynomials using simulated annealing algorithm. The method is employed to construct a freeform surface directly which is used as a primary mirror in an initial layout of an off-axis two-mirror system.
\end{abstract}

Keywords: Freeform surface, Off-axis reflection system, Two-mirror system, Seed curve extension

\section{Introduction}

Off-axis reflective optical systems are widely used in telescopes [1], hyperspectral imaging spectrometers [2], ultrashort throw ratio projection optics [3] and so on because of compact structure, having no blocking, absence of chromatic aberrations etc. [4].. However, the off-axis reflective optical systems lack rotational symmetry, which result in various asymmetrical aberrations. The freeform surfaces are generally defined as non-rotationally symmetrical optical surfaces and provide more design freedom for optical system. The asymmetry aberrations can be compensated by introducing freeform optical surfaces $[5,6]$. It is crucial for optics design to find a good initial layout as a starting point for subsequent optimization. For most of co-axis optical systems, the initial layouts can be found in some patent databases. Unfortunately, it is

\footnotetext{
* Correspondence: szp@jiangnan.edu.cn

${ }^{1}$ School of Science, Jiangnan University, Wuxi 214122, China

${ }^{2}$ Jiangsu Provincial Research Center of Light Industrial Optoelectronic Engineering and Technology, Wuxi 214122, China
}

hard to find an appropriate initial layout for an offaxis optical system from patent databases. Therefore, various methods are employed to generate the initial layout for freeform optical systems such as point-bypoint construction or construction-iteration (CI) method [7-11], the partial differential Equations (PDE) [12-15] and the Simultaneous Multiple Surface (SMS) [16-21] method. The CI method is mainly made up of two processes: construction and iteration $[8,9]$. Although the freeform surface in an off-axis reflective imaging system can be designed directly by only using the construction process [7], the image quality of the off-axis reflective system would be relatively poor if only the direct construction design process is employed [9]. To improve the image quality, an iterative process should be followed. Three iteration types including normal iteration, negative feedback and successive approximation were given in Ref. [9]. For the former two types, the iterative process could be potentially unstable in some cases. Though the iterative process of the successive 


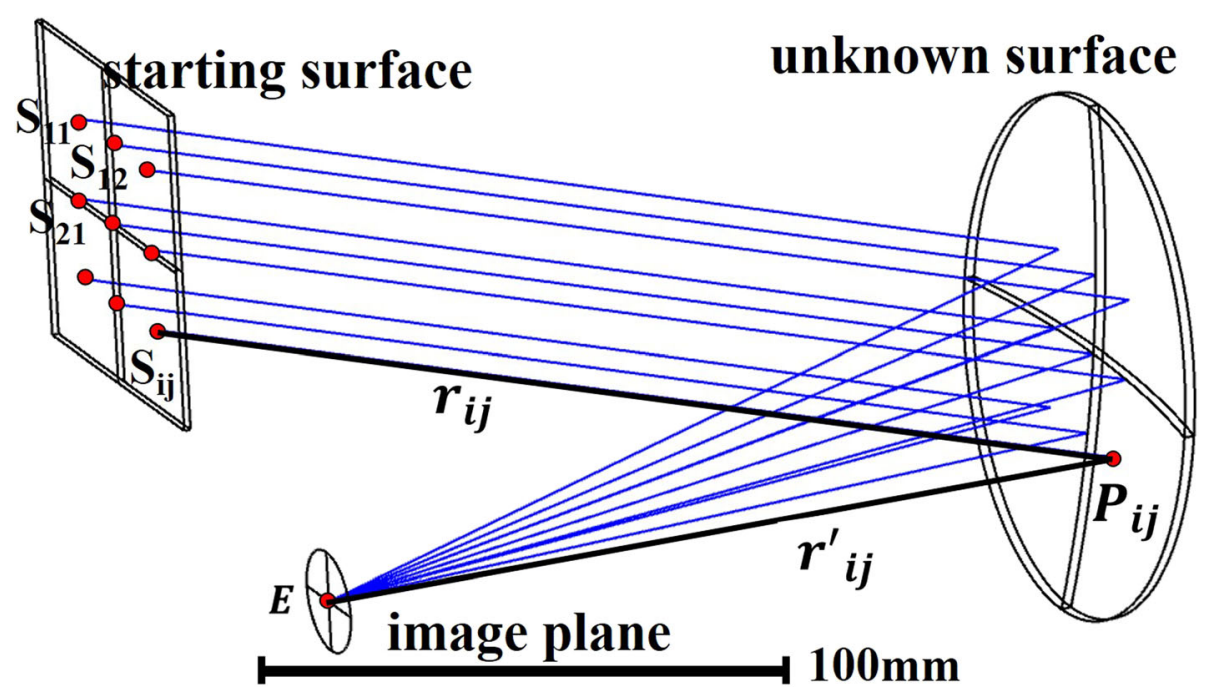

Fig. 1 The sample rays are reflected from a single freeform surface and converge to the ideal image point

approximation approach is more stable than the other two processes, the convergence is obviously slower [9]. The PDE method is to deduce a set of partial differential equations based on the mapping relationship between rays from the object and the image with some constraint conditions [12-15]. This method is generally limited by two factors: the number of surfaces and axial symmetry of the systems [12]. In Ref. [12-15], the number of designed surface is no more than two; otherwise the systems should have rotational symmetry. The SMS method was firstly proposed in non-imaging optics design [22, 23], and then it was extended to design imaging optical systems $[16,17]$. To couple properly, the SMS method generally needs the same number of optical surfaces and wave fronts, except for a few special cases. The SMS method for imaging design is mostly used in designing $2 \mathrm{D}$ cases $[16-19,21]$. The SMS method is extended to design a 3D aplanatic system in Ref. [20], which is a special case.

In this paper, the seed curve extension (SCE) method is introduced to construct the freeform surface directly in an off-axis freeform reflective image system. Before, the SCE method was only used in designing freeform surface in non-imaging optics such as LED illumination systems [24]. By the SCE method we can obtain all sample points on the unknown freeform surface. Then the continuity and smoothness of the unknown freeform
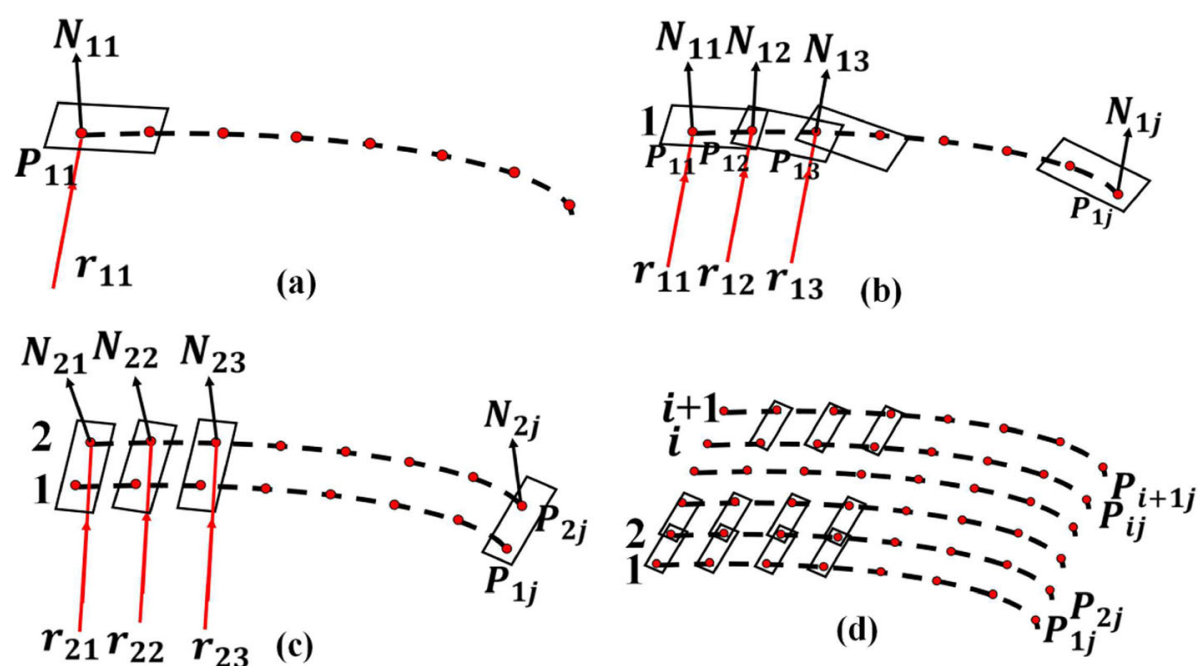

Fig. 2 Calculate the sample points on the freeform surface (a) set the first point (b) Calculate the sample points on the seed curve (c) the sample points on the curve adjacent to seed curve(d) Calculate all the sample points on the freeform surface 
surface is evaluated by calculating the angle between the normal vectors at the two adjacent points. The simulated annealing algorithm $[25,26]$ is employed to fit the sample points to an extended polynomial, which can be input into an optical software.

In order to verify the method, we constructed a freeform surface directly which is used as a primary mirror in an initial layout of an off-axis two-mirror system. In the initial layout, the secondary mirror is a planar mirror. The field of view and working F-number of the system is $2^{\circ}$ and 4.68 , respectively. The initial layout with a so designed freeform surface and a planar mirror was turned out have a good imaging quality, which can be used as a good starting point for subsequent optimization. Based on the initial layout, a further optimization is performed in Zemax. The results show that the performance of the system is diffraction-limited.

The method proposed in this paper has several improvements as follows: all sample points on unknown freeform surface can be calculated directly without iteration or feedback. Hence the speed of calculating the sample points is very fast. Also, it doesn't need to worry about the stability of the calculation process. Further, the method has no limitation in the number of the freeform surfaces or axial symmetry of the systems.

\section{Methods}

In the following, the method for constructing a freeform surface directly is proposed. The method mainly comprises four steps: (I) Set the initial and constraint condition. (II) Calculate the sample data points on the unknown freeform surface. (III) Evaluate the continuity and smoothness of the freeform surface. (IV) Fit the sample data points to an extended polynomial by simulated annealing algorithm.

(I.) Set the initial and constraint condition.

To illustrate the method of constructing freeform surface, a single freeform reflective surface is analyzed, as shown in Fig. 1. All the sample rays emitted from the starting surface. The beam is made up of $M \times M$ sample rays, whose aperture is square as shown in Fig. 1.

The space between the adjacent sample rays is equal. The starting point of the ray $r_{i j}$ is point $S_{i j}\left(X_{i j}\right.$, $\left.Y_{i j}, Z_{i j}\right)$ on the starting surface, whose coordinate is known as one of initial conditions. Also, the image point $E\left(x^{\prime}, y^{\prime}, z^{\prime}\right)$ is fixed initially. The sample rays are reflected off a single freeform surface to the ideal image point. The point $P_{i j}\left(x_{i j}, y_{i j}, z_{i j}\right)$ is the intersection of the sample ray $r_{i j}$ and the unknown surface. The rays $r_{i j}$ and $r_{i j}^{\prime}$ are called as the incident ray and the emergent ray, respectively.

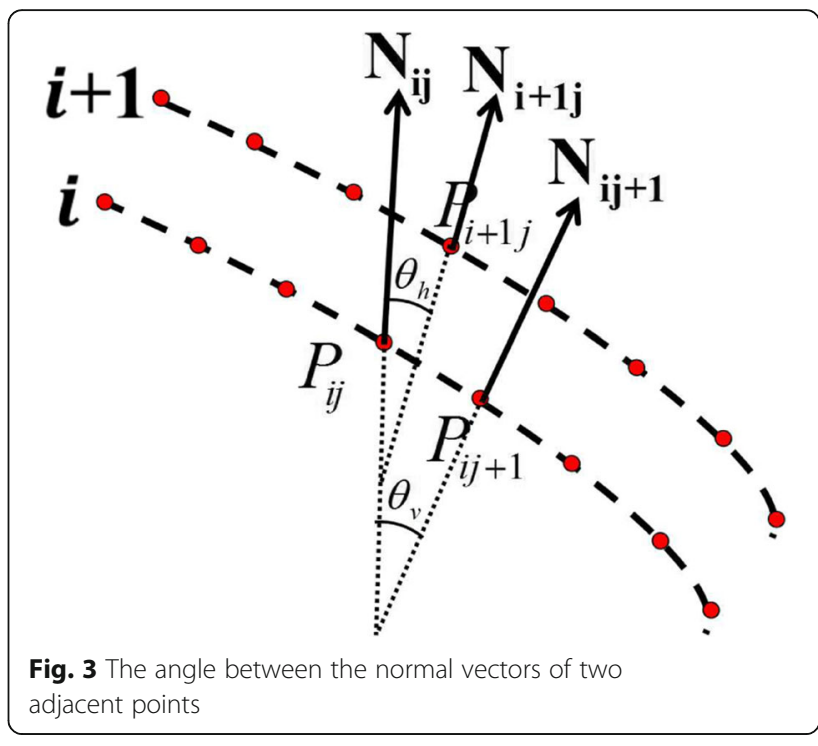

(II.)Calculate the sample points on the unknown freeform surface.

We will elaborate the process to determine the unknown freeform surface, which comprises four steps:

1) Select a point $P_{11}\left(x_{11}, y_{11}, z_{11}\right)$ along the direction of propagation for the first sample ray $r_{11}$ as the first point on the freeform surface, as shown in Fig. 2(a). The first sample ray is reflected toward the ideal image point $E\left(x^{\prime}, y^{\prime}, z^{\prime}\right)$. Thus, the first emergent and incident ray vectors are

$$
\begin{aligned}
& \mathbf{O u t}_{11}=\frac{\left(x^{\prime}-x_{11}\right) \mathbf{i}+\left(y^{\prime}-y_{11}\right) \mathbf{j}+\left(z^{\prime}-z_{11}\right) \mathbf{k}}{\sqrt{\left(x^{\prime}-x_{11}\right)^{2}+\left(y^{\prime}-y_{11}\right)^{2}+\left(z^{\prime}-z_{11}\right)^{2}}} \\
& \mathbf{I n}_{11}=\frac{\left(x_{11}-X_{11}\right) \mathbf{i}+\left(y_{11}-Y_{11}\right) \mathbf{j}+\left(z_{11}-Z_{11}\right) \mathbf{k}}{\sqrt{\left(x_{11}-X_{11}\right)^{2}+\left(y_{11}-Y_{11}\right)^{2}+\left(z_{11}-Z_{11}\right)^{2}}}
\end{aligned}
$$

Table 1 Specifications of the two-mirror system design

\begin{tabular}{ll}
\hline Parameters & Value \\
\hline Wavelength coverage & Visible-NIR(0.38-2 $\mu \mathrm{m})$ \\
Diameter of primary mirror & $100 \mathrm{~mm}$ \\
Diameter of secondary mirror & $100 \mathrm{~mm}$ \\
Effective focal length & $372.36 \mathrm{~mm}$ \\
Entrance pupil diameter & $80 \mathrm{~mm}$ \\
Full field of view & $2 \mathrm{deg}$ \\
Pixel pitch & $20 \mu \mathrm{m}$ \\
\hline
\end{tabular}




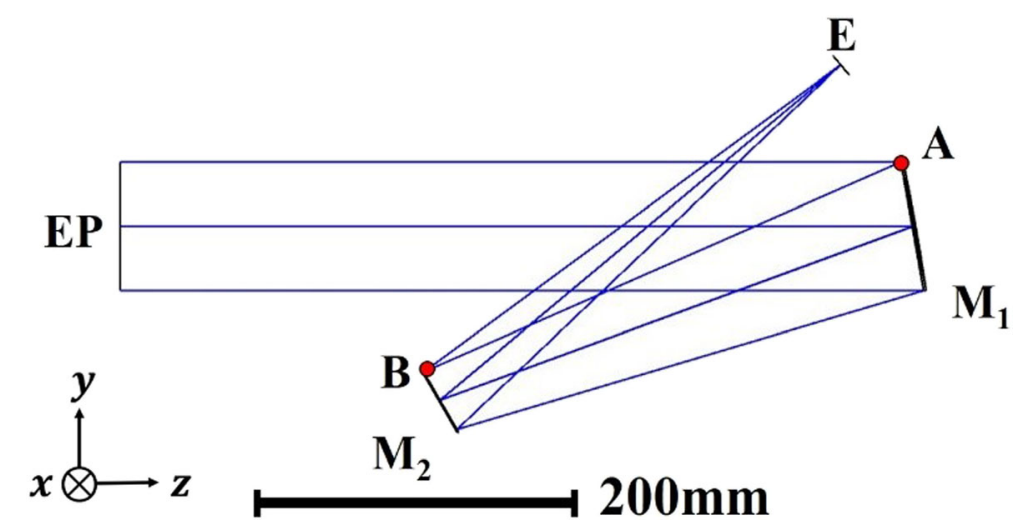

Fig. 4 Schematic of the initial layout of the two-mirror system

According to Eq. (2), the normal vector $\mathbf{N}_{11}$ of the tangent plane at the point $P_{11}$ can be obtained.

$$
\mathbf{N}_{11}=\frac{\text { Out }_{11}-\mathbf{I n}_{11}}{\sqrt{2-2\left(\mathbf{O u t}_{11} \cdot \mathbf{I n}_{11}\right)}}
$$

2) Determining the next point $P_{12}$ on the freeform surface, which is the intersection of the adjacent sample ray $r_{12}$ and the tangent plane at $P_{11}$, whose normal vector is $\mathbf{N}_{11}$. The sample ray $r_{12}$ is emitted from $S_{12}$, as presented in Fig. 1. Repeat the above procedure for other sample rays $r_{1 j}$ on the first row consecutively, and the intersection of the first column of sample rays $r_{1 j}$ and the unknown freeform surface can be sequentially calculated, as shown in Fig. 2(b). Hence we obtain the first curve on the freeform surface, which we shall refer to as the seed curve, which is Curve 1 in Fig. 2(b).

3) After the seed curve, Curve 1 is determined, we return to the initial point $P_{11}$ to start calculating the next curve on the freeform surface, Curve 2 as shown in Fig. 2(c). The point $P_{21}$ is the intersection of the sample ray $r_{21}$ and the tangent plane at point $P_{11}$, as presented in Fig. 2(c). Similarly, we can calculate all the other sample data points along Curve 2, by locating the intersection of the sample rays $r_{2 j}$ on the second row and the tangent planes at the corresponding points along Curve 1 .

4) By repeating Step (3), we can build the recursive relationships between the corresponding points on Curve $i$ and Curve $i+1$. Based on the recursive relationships, all the other sample points on the freeform surface can be determined, as shown in Fig. 2(d).

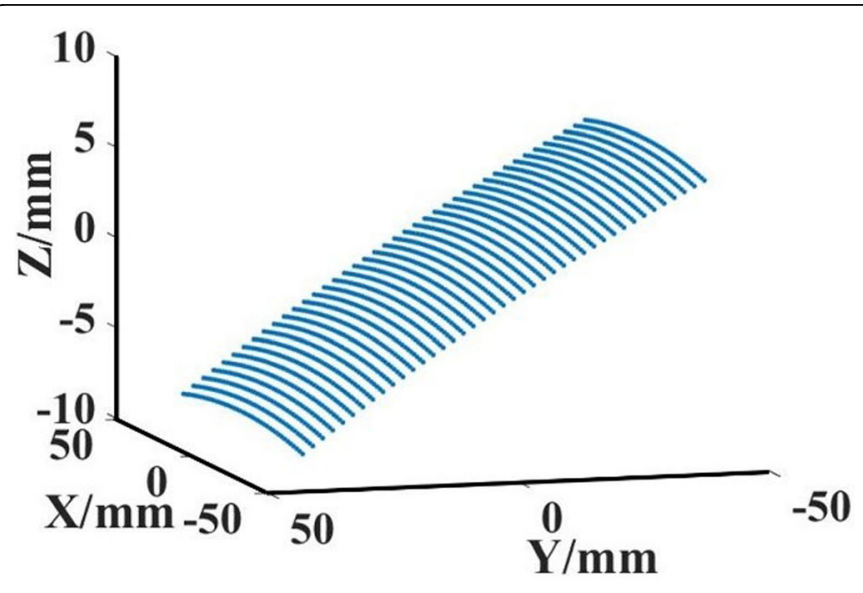

(a)

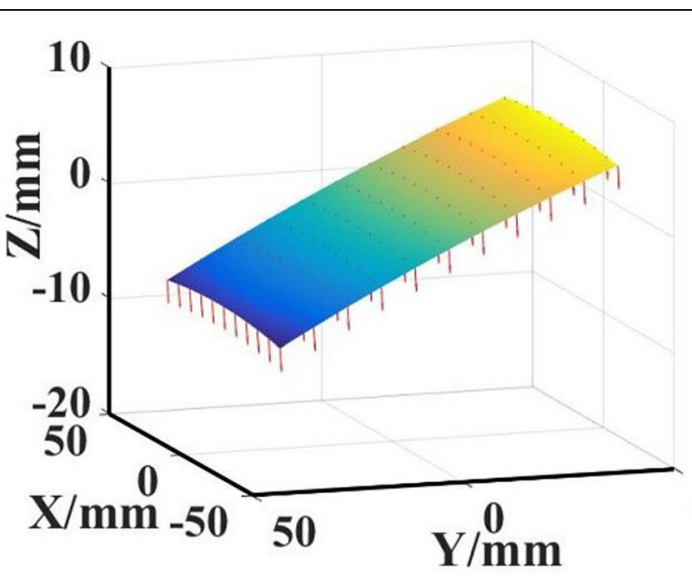

(b)

Fig. 5 (a) The sample points on the freeform surface; (b) The normal vector at each sample point 


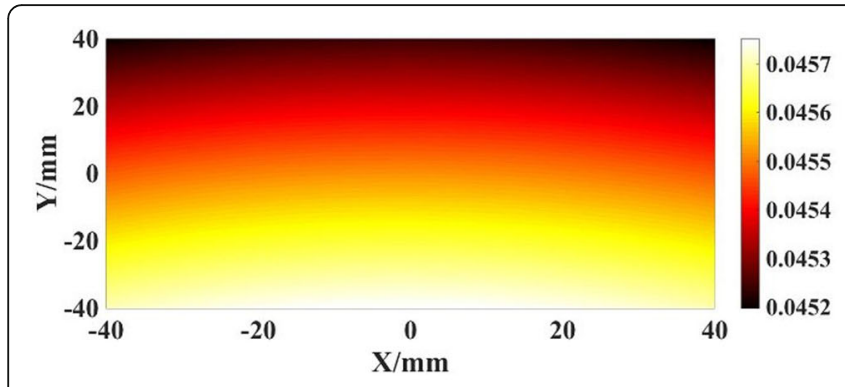

(a)

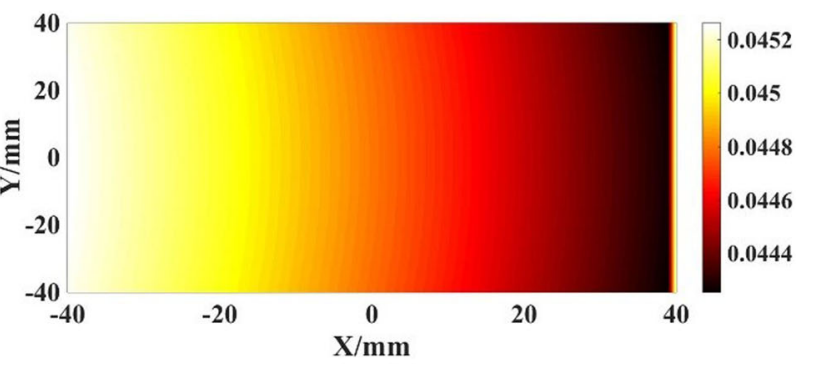

(b)

Fig. 6 (a) The angle between the normal vectors of two adjacent points along the same curve; (b) the angle between the normal vectors of two corresponding points along the adjacent curve

(III.Evaluate the continuity and smoothness of the freeform surface.

All the sample points can be obtained by the method above. Actually, one needs to construct a smooth and continuous freeform surface according to the discrete sample points. Therefore, the continuity of the freeform surface was evaluated by the following method. The angle between the normal vectors of two adjacent points is shown in Fig. 3, which can be calculated by Eq. (3) [24]. The $\theta_{\mathrm{v}}$ is the angle between the normal vectors of two adjacent points along the same curve. The $\theta_{\mathrm{h}}$ is the angle between the normal vectors of two corresponding points along the adjacent curves. In addition to the angles, the space between the adjacent sample points and the gradient of all sample points on the unknown freeform surface are also key to evaluate the continuity and smoothness.

$$
\theta=\arccos \left(\frac{\mathbf{N}_{1} \cdot \mathbf{N}_{3}}{\left|\mathbf{N}_{1}\right| \times\left|\mathbf{N}_{3}\right|}\right)
$$

(IV.Fit the sample data points to an extended polynomial.

The previous design basing on seed curve extension algorithm is done for a single field point. In order to further optimize the freeform surface to cover a larger field of view, the freeform surface has to be expressed by a set of appropriate polynomials. The coefficients of these polynomials can be further optimized with a commercial optical design software, such as Zemax. There are a number of polynomials that can be used as analytical expressions of the freeform surface, such as the Zernike polynomials, the Chebyshev polynomials, and the extended polynomials. In this paper, the XY extended polynomials are employed to describe the freeform surface. The optical system in this paper is symmetrical about the YOZ plane, therefore, only even-order terms in $\mathrm{x}$ are used in the XY polynomials. A base conic surface and 11 added polynomial aspheric terms up to fifth order are used. The surface sag is of the form $[1,7]$ :

$$
\begin{aligned}
& z=\mathrm{f}(x, y) \\
& \mathrm{f}(\mathrm{x}, \mathrm{y})=\frac{c\left(x^{2}+y^{2}\right)}{1+\sqrt{1-(1+k) c^{2}\left(x^{2}+y^{2}\right)}}+A_{2} y+A_{3} x^{2}+A_{5} y^{2}+A_{7} x^{2} y \\
& +A_{9} y^{3}+A_{10} x^{4}+A_{12} x^{2} y^{2}+A_{14} y^{4}+A_{16} x^{4} y+A_{18} x^{2} y^{2}+A_{20} y^{5}
\end{aligned}
$$

Where $c$ is the curvature of the base conic surface, $k$ is the conic coefficient, and $A_{q}$ is the q-th polynomial coefficient. In order to find the optimal fitting parameters $\left(c, k, A_{q}\right)$, an evaluation function is constructed as Eq. (5).

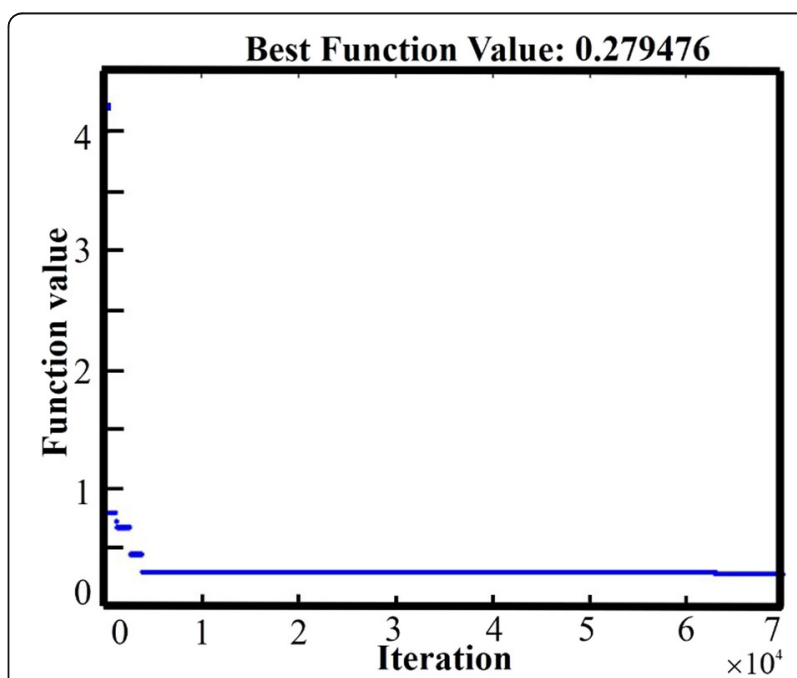

Fig. 7 The dependence of the evaluation function on the iteration numbers 
Table 2 The optimal fitting parameters

\begin{tabular}{llll}
\hline Parameters on & Values & Parameters on & Values \\
\hline$c$ & $-8.069 \mathrm{e}-04$ & $X^{4}$ & -0.046 \\
$k$ & -8.049 & $X^{2} Y^{2}$ & -0.09191 \\
$Y$ & -17.75 & $Y^{4}$ & -0.046 \\
$X^{2}$ & $-1.553 \mathrm{e}-05$ & $X^{4} Y$ & $-2.085 \mathrm{e}-03$ \\
$Y^{2}$ & $4.44 \mathrm{e}-04$ & $X^{2} Y^{3}$ & $-1.185 \mathrm{e}-03$ \\
$X^{2} Y$ & $2.515 \mathrm{e}-04$ & $Y^{5}$ & $-9.392 \mathrm{e}-04$ \\
$Y^{3}$ & $1.644 \mathrm{e}-04$ & & \\
\hline
\end{tabular}

$$
F\left(c, k, A_{q}\right)=\sqrt{\frac{\sum_{i=1}^{M} \sum_{j=1}^{M}\left(z_{i, j}-f\left(x_{i}, y_{j}\right)\right)^{2}}{M \times M}}
$$

Where $z_{i}$ is the z-coordinates of any sample point $\left(x_{i}\right.$, $\left.y_{j}, z_{i, j}\right)$. And $f\left(x_{i}, y_{j}\right)$ is calculated according to Eq. (4) .It is clearly that $\left(c, k, A_{q}\right)$ are the independent variables of the evaluation function. As known, when the evaluation function reaches the minimum, the fitting accuracy is the best. The minimum value of evaluation function is found by the simulated annealing algorithm $[25,26]$. Once the minimum value of evaluation function is obtained, the optimal fitting parameters also obtained.

\section{Results and discussion}

In order to verify our method, an initial layout of an off-axis two-mirror system with one freeform surface is designed. The specifications of the freeform two-mirror system are listed in Table 1, which is given in Ref. [1]. In the initial layout of the off-axis two-mirror system, the primary mirror $M_{1}$ is a freeform surface, and the secondary mirror $M_{2}$ is a planar mirror as shown in Fig. 4. The EP is the entrance pupil, which is also the starting plane of all sample rays. The entrance pupil diameter of the system is $80 \mathrm{~mm}$ and the sample beam aperture is rectangular. The rectangular aperture can be cut into a circular aperture at the last. Point $\mathrm{A}$ is the first point on the freeform surface, which is set at begin. Point $\mathrm{B}$ is the first point on the secondary mirror which is titled 60 degree about $\mathrm{z}$-axis. The distance between point $A$ and point $B$ is $300 \mathrm{~mm}$ away. The sample rays are reflected by the primary mirror and the secondary mirror and converge into an ideal image point as shown in Fig. 4.

The spacing of adjacent sample rays along the horizontal and vertical directions are both chosen to be 0.5 $\mathrm{mm}$, and a total $\mathrm{K}=161 \times 161$ sample rays are employed for ray tracing.

According to the SCE method, the sample points on freeform surface are obtained as shown in Fig. 5(a). The normal vector at every sample point is shown in Fig. 5(b). It is important to ensure continuity of the freeform surface for the purpose of manufacture on freeform surface mirror. In order to evaluate the continuity of the freeform surface, we calculate the angle between the normal vectors of two adjacent points by Eq. (3). Figure 6 (a) and (b) show the values of the angle between the normal vectors of two adjacent points. Figure 6 (a) is the angle between the normal vectors of two adjacent points along the same curve. Figure 6 (b) is the angle between the normal vectors of two corresponding points along the adjacent curve. The spaces of the adjacent sample points along $\mathrm{x}$ and $\mathrm{y}$ direction are both $0.5 \mathrm{~mm}$. It is shown that the maximum angle between the normal vectors of two adjacent points is 0.0457 degree, which is quite small. In addition, the maximum gradient of all the sample points are 0.2 . Then, we make the gradient calculation again. The calculation show that all the gradient values are almost near zero. Therefore, the continuity of the freeform surface is very good.

Here, the sample data points are fitted to an extended polynomial as shown in Eq. (4). In order to obtain the optimal fitting coefficient, an evaluation function is constructed as Eq. (5), which reflects the fitting accuracy. When the evaluation function reaches the minimum, the

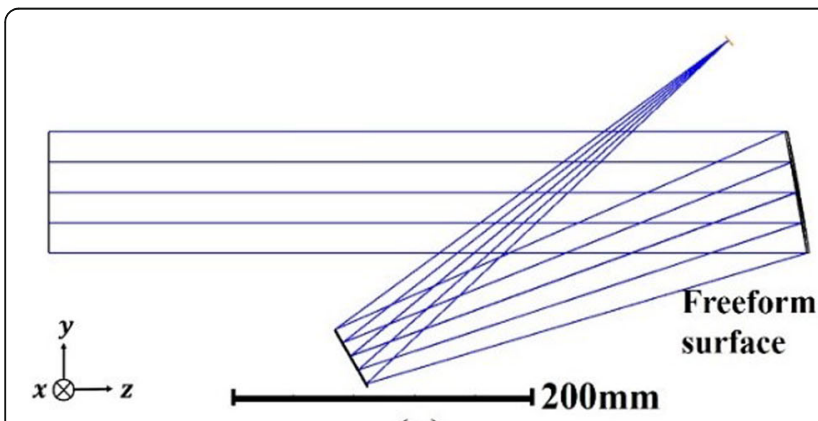

(a)

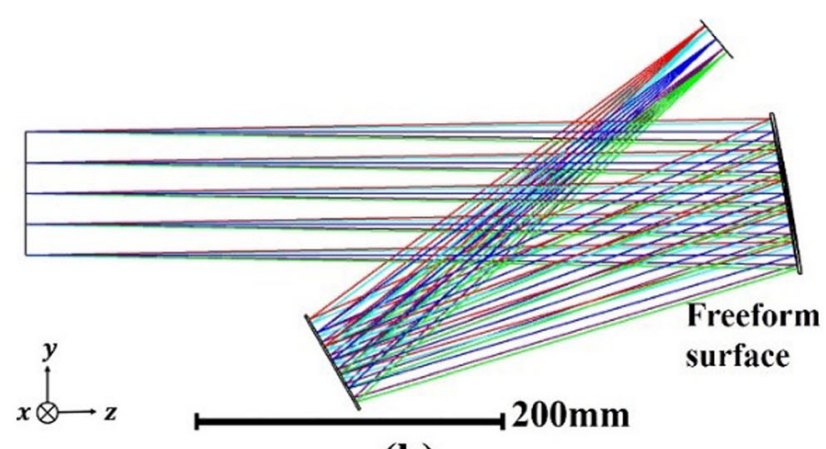

(b)

Fig. 8 The initial layout with a single field of view (a) and full field of view (b) 


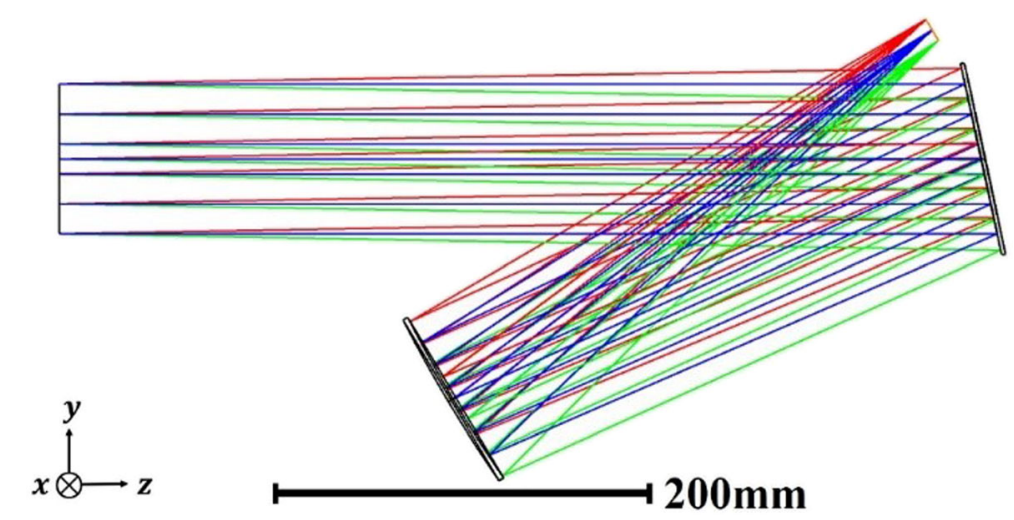

Fig. 9 The optimized system layout with multi-field of view

fitting parameters are optimal. The simulated annealing algorithm is employed to seek the minimum of the evaluation function. Figure 7 shows the dependence of evaluation function on the iteration numbers. The optimal parameters are listed in Table 2.

Then the two-mirror system with a freeform and a planar mirror can be imported into Zemax. Figure 8 (a) shows the optical system layout with a central field of view. The RMS radius of the spot diagram at central field of view is $2.573 \mu \mathrm{m}$. Figure 8 (b) shows that more field points are added to the initial system based on the system specification requirements. The average RMS radius of all the spot diagrams at the full fields of view for the initial system is $95.96 \mu \mathrm{m}$.

Although the performance of initial layout is decreasing after adding more field points, it can be used as a good starting point for further optimization of the system with Zemax. Basing on the starting point, the final freeform off- axis two-mirror system can be quickly obtained through optical software optimization as shown in Fig. 9. In the optimization, the spacing between the two mirrors are chosen to be fixed. The secondary mirror was chosen to be a conic surface during optimization. The MTF plots of the final optical system are shown in Fig. 10. The MTFs of all the field points are greater than 0.7 at $25 \mathrm{lp} / \mathrm{mm}$, which is the Nyquist frequency. It is obvious that the performance of the system is near the diffraction limit at Visible-NIR $(0.38-2 \mu \mathrm{m})$. The average RMS radius of all the spot diagrams at the full fields of view for the final system is $4.03 \mu \mathrm{m}$ as shown in Fig. 11 and the maximum grid distortion is $-0.14 \%$ as shown in Fig. 12. In this design, the primary mirror is a freeform surface, and the secondary mirror is a conic surface.

Clearly, by the SCE method, while the initial point is set, all other points on unkown freeform surface can be obtained based on recursive relationship. So each point

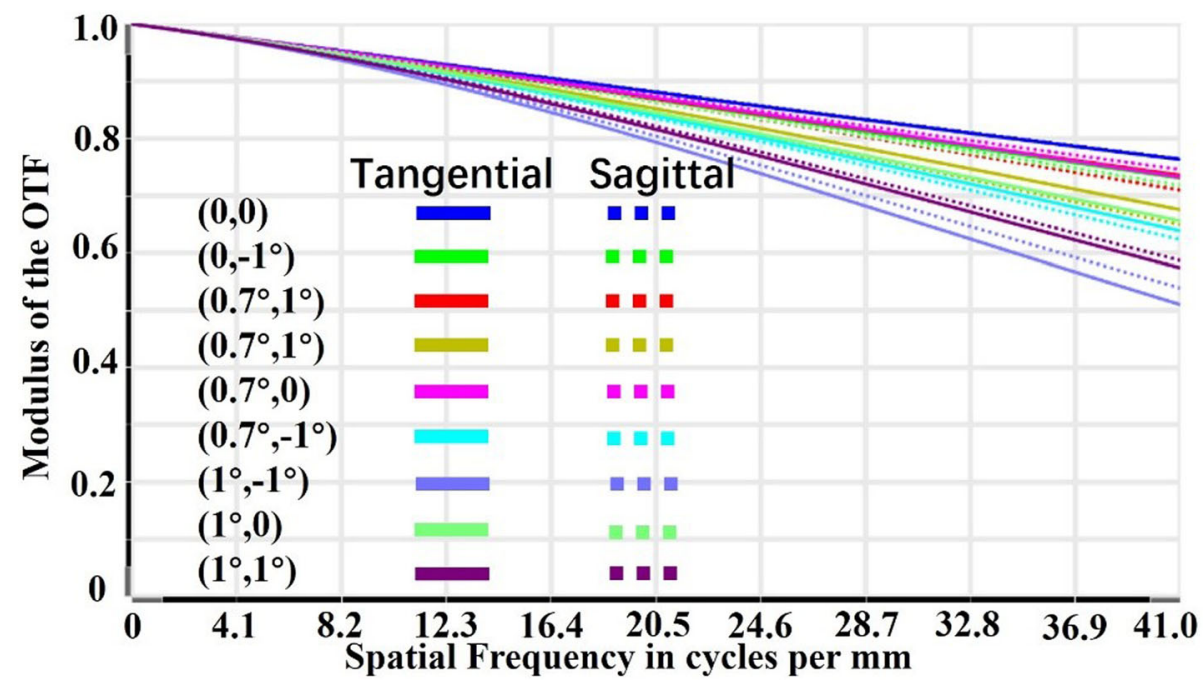

Fig. 10 The MTF plots of the final system at different fields of view 


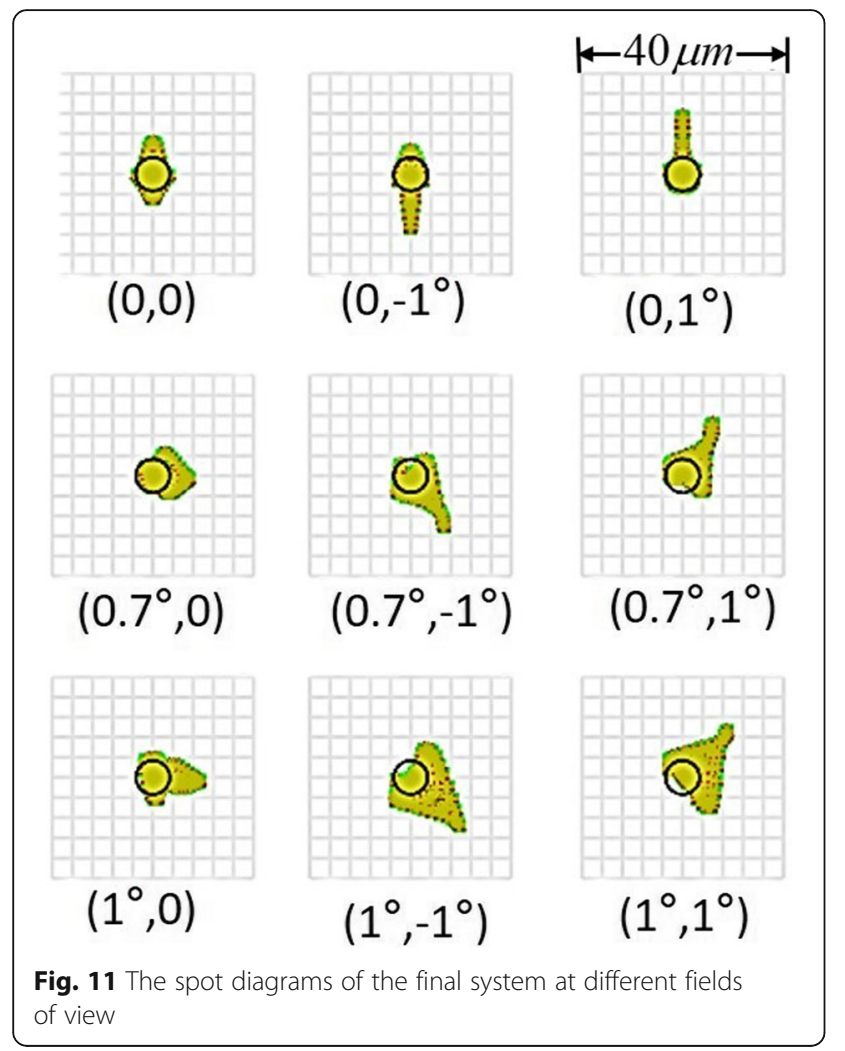

on the initial freeform surface can be obtained at once. In other words, the positions of each point on the initial freeform surface is obtained directly without iteration or feedback. This leads to two obvious advantages: (1) the speed of positioning the sample points is very fast; (2) the method obviates the consideration of whether there is a solution or the stability of the solution. In addition,

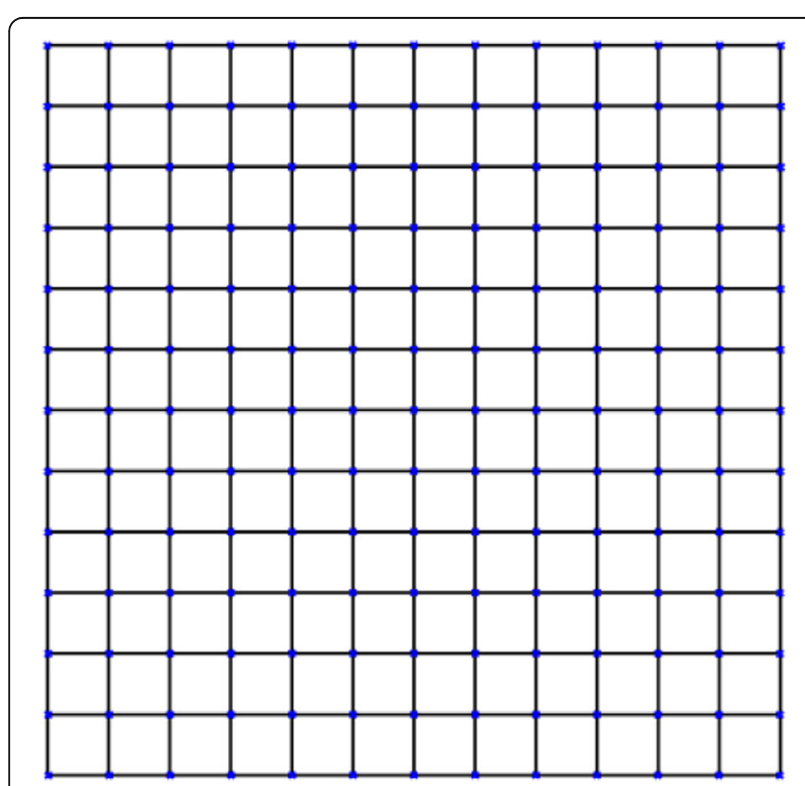

Fig. 12 Grid distortion the method has no limitation in the number of the freeform surfaces. Although the two-mirror system with one freeform surface is shown as an example, the method can be extended to design off-axis systems with more freeform surfaces by adding a virtual image point to each surface, which will be discussed in future works.

\section{Conclusion}

In conclusion, a method is introduced to construct a freeform surface directly in off-axis reflective image system. The method is a combination of seed curve extension and simulated annealing algorithm. The seed curve extension algorithm can calculate the sample points quickly without worrying about the stability of the calculating process. The sample points can be fitted to an extended polynomials by simulated annealing algorithm. The method is employed to construct a freeform surface directly, which is used as a primary mirror in an initial layout of an off-axis two-mirror system. The field of view and working F-number of the system is $2^{\circ}$ and 4.68 , respectively. The initial layout is made up of one freeform surface and one planar surface, whose RMS radius of the spot diagram at central field of view is $2.573 \mu \mathrm{m}$. Based on the initial layout, a further optimization is performed in Zemax. The results show that the performance of the system is diffraction- limited at Visible-NIR $(0.38-2 \mu m)$.

\section{Acknowledgements}

Not applicable.

\section{Authors' contributions}

All authors read and approved the final manuscript.

\section{Funding}

Not applicable.

Availability of data and materials

Detail about data has been provided in the article.

\section{Competing interests}

The authors declare that they have no competing interests.

Received: 15 June 2019 Accepted: 31 March 2020

Published online: 19 April 2020

\section{References}

1. Gautam, S., Gupta, A., Singh, G.S.: Optical design of off-axis Cassegrain telescope using freeform surface at the secondary mirror. Optical Engineering. 54, 025113 (2015)

2. Chang, L., Christoph, S., Thomas, F.P., Zeitner, U.D., Herbert, G.: Comparison of hyperspectral imaging spectrometer designs and the improvement of system performance with freeform surfaces. Appl. Opt. 56, 6894-6901 (2017)

3. Nie, Y., Mohedano, R., Benítez, P., Chaves, J., Miñano, J., Thienpont, H., Duerr, F.: Multifield direct design method for ultrashort throw ratio projection optics with two tailored mirrors. Appl. Opt. 55, 3794-3800 (2016)

4. Stewart, W.: Freeform Optics: Notes from the Revolution. Optics Photonics News. 28, 34-41 (2017)

5. Shi, H., Jiang, H., Zhang, X., Wang, C., Liu, T.: Analysis of nodal aberration properties in off-axis freeform system design. Appl. Opt. 55, 6782-6790 (2016) 
6. Bauer, A., Rolland, J.P., Thompson, K.P.: Ray-based optical design tool for freeform optics: coma full-field display. Opt. Express. 24, 459-472 (2016)

7. Yang, T., Zhu, J., Hou, W., Jin, G.: Design method of freeform off-axis reflective imaging systems with a direct construction process. Opt. Express. 22, 9193-9205 (2014)

8. Yang, T., Zhu, J., Jin, G.: Design of freeform imaging systems with linear field-of-view using a construction and iteration process. Opt. Express. 22, 3362-3374 (2014)

9. Yang, T., Zhu, J., Wu, X., Jin, G.: Direct design of freeform surfaces and freeform imaging systems with a point-by-point three-dimensional construction-iteration method. Opt. Express. 23, 10233-10246 (2015)

10. Gong, T., Jin, G., Zhu, J.: Point-by-point design method for mixed-surfacetype off-axis reflective imaging systems with spherical, aspheric, and freeform surfaces. Opt. Express. 25, 10663-10676 (2017)

11. Zhu, J., Yang, $T$., Jin, G.: Design method of surface contour for a freeform lens with wide linear field-of-view. Opt. Express. 21, 26080-26092 (2013)

12. Volatier, J.B., Druart, G.: Differential method for freeform optics applied to two-mirror off-axis telescope design. Opt. Lett. 44, 1174-1177 (2019)

13. Liu, J., Benítez, P., Miñano, J.C.: Single freeform surface imaging design with unconstrained object to image mapping. Opt. Express. 22, 30538-30546 (2014)

14. Zhu, J., Wu, X., Yang, T., Jin, G.: Generating optical freeform surfaces considering both coordinates and normals of discrete data points. J. Opt. Soc. Am. A. 31, 2401-2408 (2014)

15. Bian, Y., Li, H., Wang, Y., Zheng, Z., Liu, X.: Method to design two aspheric surfaces for a wide field of view imaging system with low distortion. Appl. Opt. 54, 8241-8247 (2015)

16. Benítez, P., Miñano, J.C.: Ultrahigh-numerical-aperture imaging concentrator. J. Opt. Soc. Am. A. 14, 1988-1997 (1997)

17. Miñano, J., Benítez, P., Lin, W., Infante, J., Muñoz, F., Santamaría, A.: An application of the SMS method for imaging designs. Opt. Express. 17, 24036-24044 (2009)

18. Duerr, F., Benítez, P., Miñano, J., Meuret, Y., Thienpont, H.: Analytic design method for optimal imaging: coupling three ray sets using two free-form lens profiles. Opt. Express. 20, 5576-5585 (2012)

19. Lin, W., Benítez, P., Miñano, J.C., Infante, M.J., Biot, G., Marta, D.F.L.: SMS based optimization strategy for ultra-compact SWIR telephoto lens design. Opt. Express. 20, 9726-9735 (2012)

20. Miñano, J., Benítez, P., Narasimhan, B.: Freeform aplanatic systems as a limiting case of SMS. Opt. Express. 24, 13173-13178 (2016)

21. Nie, Y., Duerr, F., Thienpont, H.: Direct design approach to calculate a twosurface lens with an entrance pupil for application in wide field-of-view imaging. Opt. Eng. 54, 015102 (2015)

22. Winston, R., Miñano, J.C., Benítez, P., Shatz, N., Bortz, J.: Nonimaging Optics. Academic Press Elsevier. 181-189 (2004)

23. Chaves, J.: Introduction to Nonimaging Optics. CRC Press. 271-299 (2008)

24. Kai, W., Sheng, L., Fei, C., Zong, Q., Zongyuan, L., Xiaobing, L.: Freeform LED lens for rectangularly prescribed illumination. J. Opt. A: Pure Appl. Opt. 11, 105501 (2009)

25. Su, Z., Xue, D., Ji, Z:: Designing LED array for uniform illumination distribution by simulated annealing algorithm. Opt. Express. 20, A843-A855 (2012)

26. Kirkpatrick, S., Gelatt, C.D., Vecchi, M.P.: Optimization by Simulated Annealing. Science. 220, 671-680 (1983)

\section{Publisher's Note}

Springer Nature remains neutral with regard to jurisdictional claims in published maps and institutional affiliations.

\section{Submit your manuscript to a SpringerOpen ${ }^{\circ}$ journal and benefit from:}

- Convenient online submission

- Rigorous peer review

- Open access: articles freely available online

- High visibility within the field

- Retaining the copyright to your article

Submit your next manuscript at $\boldsymbol{\nabla}$ springeropen.com 